\title{
The Test of a New Critical Exponent $Q$ by Using Ising Model on the Creutz Cellular Automaton
}

\author{
Z. Merdan* And D. GokBel-KeKlikoglu \\ Gazi University, Faculty of Science, Department of Physics, 06500 Teknikokullar, Ankara, Turkey \\ (Received July 10, 2017; in final form January 2, 2018)
}

\begin{abstract}
Above the upper critical dimension $d_{c}$ the Ising model is simulated on the Creutz cellular automaton. The values of a new critical exponent $\mathbf{Q}$ are obtained by using the simulations for the order parameter and the magnetic susceptibility. At $d=4,5,6,7,8$, the values of the new critical exponent $Q$ are $0.9904(16), 1.2721(2)$, $1.4806(24), 1.7626(17), 1.9997(50)$ for the order parameter, respectively, while those 1.0415(13), 1.2987(27), $1.5133(1), 1.7741(1), 2.0133(28)$ are for the magnetic susceptibility in the same order. The computed values of the new critical exponent $\mathbf{Q}$ are in agreement with theoretical values.
\end{abstract}

DOI: 10.12693/APhysPolA.133.1200

PACS/topics: Ising model, cellular automata, critical exponents, finite-size scaling, modified finite-size scaling

\section{Introduction}

The effect of dimensionality on phase transitions should be investigated because the $d$-dimensional Ising model is not directly applicable to real magnetic systems [1]. In fact, in Euclidean quantum field theory, the 4D Ising model describes the physical dimension. In higher dimension and/or higher lattice size, the simulation of the Ising model by the conventional Monte Carlo method becomes impractical and the algorithms need to be faster. The Creutz cellular automaton [2,3] does not require high-quality random numbers, it is an order of magnitude faster than the conventional Monte Carlo method and compared to the Q2R cellular automaton [4], it has the advantage of fluctuating internal energy from which the specific heat can be computed.

It is well known that standard form of the hyperscaling relation, i.e., $\nu d=2-\alpha$, is valid at and below the upper critical dimension $d=d_{c}$; however, it fails above $d_{c}=4$. Thus, it needs to be revised and it can be rewritten in the following form [5]:

$$
\frac{\nu d}{\mathrm{Q}}=2-\alpha
$$

Here, the general hyperscaling relation is obtained for all dimensions by the help of a new critical exponent $Q$ ("koppa" $[5,6]$ ). The exponent $Q$ is considered to be 1 at $d \leq d_{c}$ because the standard hyperscaling relation is valid there. This relation can be also represented by $\nu d_{c}=2-\alpha$ because it is valid for $d=d_{c}$. As Eq. (1) is considered, it is obviously seen that $\mathrm{Q}=d / d_{c}$. This exponent is also universal as the other exponents.

The conventional form for finite-size scaling (FSS) is transformed above the upper critical dimension $d=d_{c}$. Let $P_{L}(t)$ represent an observable $P$ measured for a system of linear extent $L$ at reduced temperature $t$. If

\footnotetext{
*corresponding author; e-mail: ziyamerdan@gazi.edu.tr
}

$P_{\infty}(t) \sim|t|^{-\rho}$, conventional FSS posits that $P_{L}(t) \sim$ $L^{\rho / \nu}$ inside the scaling window $[5,7]$. Above $d_{c}$, however, this conventional form is replaced by

$$
P_{L}(t) \sim L^{\frac{Q_{\rho}}{\nu}}
$$

This is called the modified finite-size scaling (Q-FSS) to distinguish it from the conventional form $[6,8,9]$. Thus, Q-FSS contains information on the exponent $Q$ and can be used to measure it.

The purpose of this study is to test Q-FSS relations for the order parameter and the magnetic susceptibility near the infinite-lattice critical temperature in $d \geq 4$ dimensions on the Creutz cellular automaton. The new critical exponent $Q$ for the order parameter and the magnetic susceptibility are obtained by analyzing the data from the simulations of the Ising model on the Creutz cellular automaton.

The simulations are carried out on the Creutz cellular automaton $[2,10]$ which has arisen as an alternative research tool for the Ising model investigations and has simulated the Ising model in the dimensionalities $4 \leq d \leq 8[11,12]$.

This paper is organized as follows. The model is described in Sect. 2, the results are discussed in Sect. 3, and a conclusion is given in Sect. 4.

\section{Model}

The algorithm is readily generalized to any dimension or other lattice structures. In general, $n$ binary bits are associated with each site of the lattice. The value for each site is determined from its value and from those of its nearest neighbors at the previous time step. The updating rule, which defines a deterministic cellular automaton, is as follows: of the $n$ binary bits on each site, the first one is the Ising spin $B_{i}$. Its value may be " 0 " or "1". The Ising spin energy, or internal energy of the lattice, $H_{I}$, is given (in units of the nearest neighbor coupling constant $J)$ by $[2,3,10,11]$ : 


$$
H_{I}=-\sum_{\langle i, j\rangle} S_{i} S_{j}
$$

where $S_{i}=2 B_{i}-1$ and $\langle i j\rangle$ denotes the sum over all nearest neighbor pairs of sites.

The next $n-2$ bits on each site represent the demon or momentum variable conjugated to the spin. These $n-2$ bits form an integer which can take on the values within the interval $\left(0, N=\sum_{i=1}^{n-2} 2^{i-1}\right)$. Denoting these bits by $D_{1 i}, D_{2 i}, \ldots, D_{n i}$, we associate with them the kinetic energy

$$
H_{K}=4 \sum_{i}\left(D_{1 i} x 2^{0}+D_{2 i} x 2^{1}+\ldots+D_{n i} x 2^{n-1}\right)
$$

which is conserved; here $H_{K}$ is the kinetic energy of the lattice. For a given total energy the system temperature $T$ (in units of $J / k_{\mathrm{B}}$ where $k_{\mathrm{B}}$ is the Boltzmann constant and $m$ is mass) is obtained from the average value of the kinetic energy of a demon,

$$
\left\langle E_{D}\right\rangle=\frac{\sum_{m=0}^{N}(4 m) \mathrm{e}^{(-4 m) / T}}{\sum_{m=0}^{N} \mathrm{e}^{(-4 m) / T}} .
$$

The $n$-th bit associated with each site provides a checkerboard style updating because this bit gives the space-time parity of the site. A checkerboard style updating aims to get rid of the result of stating that any cellular automaton rule which updates all spins simultaneously cannot simulate the Ising model and so it allows the simulations of the Ising model on a cellular automaton. The black sites of the checkerboard are updated and then their color is changed into white; the white sites are changed into black without being updated and vice versa.

The updating rules for the spin and the momentum variables are as follows: for a site to be updated its spin is flipped and the change in the Ising energy (internal energy) $H_{\mathrm{I}}$, is calculated. If this energy change is transferable to or from the momentum variable associated with this site, such that the total energy $H$ is conserved, then this change is done and the momentum is appropriately changed. Otherwise the spin and the momentum are not changed.

As the initial configuration, all the spins are taken ordered (up or down). The initial kinetic energy is given to the lattice via the appropriate bits of the momentum variables in the white sites randomly.

The simulations are carried out on simple hypercubic lattices $L^{d}$ of dimensionality $d$ and linear dimension $L$ with periodic boundary conditions.

\section{Results and discussion}

The Privman-Fisher hypothesis for the singular part of the free-energy density $f_{L}^{(S)}(t, h)$ of a hypercubic finite system $L^{d}$ with periodic boundary conditions is adapted for the Ising model in the critical dimension $d_{C}=4$, by proposing the finite-size scaling function $Y(x . y)$, correct to leading logarithms as below [13]:

$$
\begin{aligned}
& f_{L}^{(S)}(t, h)=L^{-d} Y\left(t L^{2} \log ^{1 / 6} L, h L^{3} \log ^{1 / 4} L\right), \\
& t \rightarrow 0, \quad h \rightarrow 0, \quad L \rightarrow \infty,
\end{aligned}
$$

where $t=\left(T-T_{c}\right) / T_{c}$ is the reduced temperature and $h$ is the external magnetic field. The order parameter $M_{L}(t, h)$ and the magnetic susceptibility $\chi_{L}(t, h)$ are obtained from Eq. (6) as below [13]:

$$
\begin{aligned}
& M_{L}(t, h)=-\frac{\partial f_{L}}{\partial h}= \\
& \quad L^{-\frac{\beta}{\nu} \boldsymbol{Q}} \log ^{1 / 4}(L) U\left(t L^{2} \log ^{1 / 6} L, h L^{3} \log ^{1 / 4} L\right), \\
& \chi_{L}(t, h)=-\frac{\partial^{2} f_{L}}{\partial h^{2}}= \\
& \quad L^{\frac{\gamma}{\nu}} \mathbf{Q} \log ^{1 / 2}(L) V\left(t L^{2} \log ^{1 / 6} L, h L^{3} \log ^{1 / 4} L\right),
\end{aligned}
$$

where $U, V$ are the corresponding finite size scaling functions. For $h=0$ and at $T=T_{c}$, they become the following equations with a new critical exponent $\mathbf{Q}$ :

$$
\begin{aligned}
& M_{L}(t)=L^{\frac{-\beta Q}{\nu}} \log ^{1 / 4} L, \\
& \chi_{L}(t)=L^{\frac{\gamma P}{\nu}} \log ^{1 / 2} L .
\end{aligned}
$$

At the critical temperature, the values of the order parameter and the magnetic susceptibility are determined by using the data obtained from the simulations of the Ising model on the Creutz cellular automaton. With these values, the graphs are plotted for the order parameter and the magnetic susceptibility by using Eq. (8) and Eq. (9) as seen in Fig. 1. The slopes of the graphs are $-\beta \mathbf{Q} / \nu$ and $\Upsilon \mathbf{Q} / \nu$, respectively. Since the Ising model
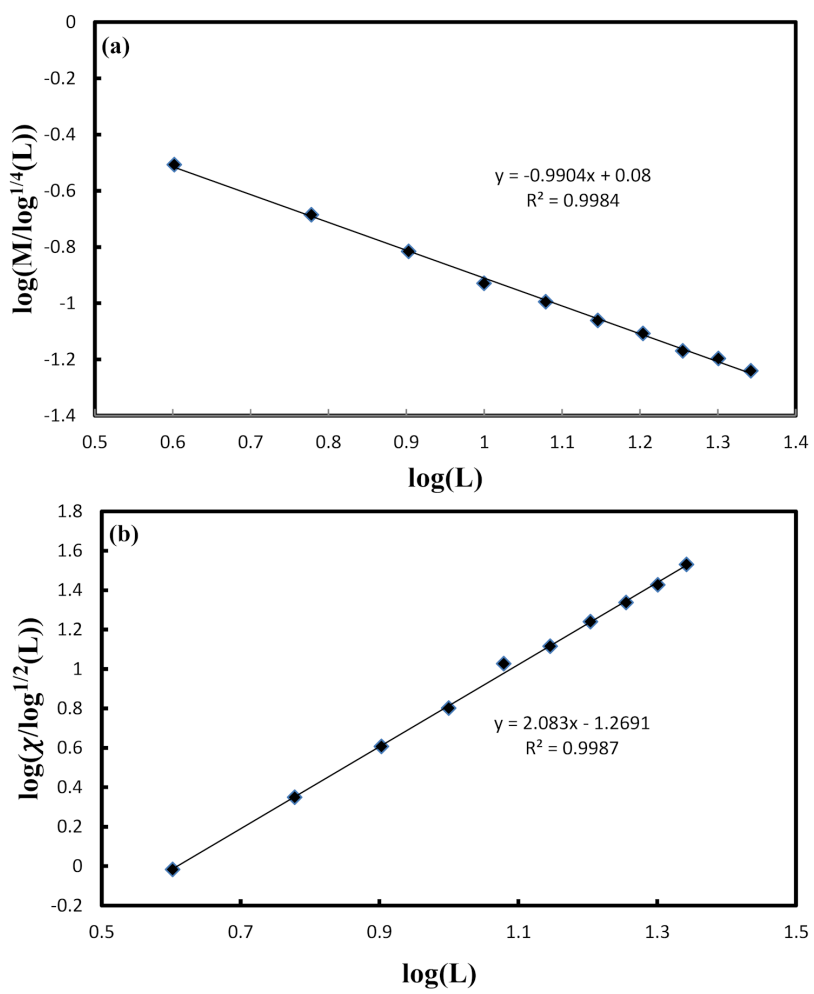

Fig. 1. The modified finite-size scaling (Q-FSS) for $d=4$ : (a) the order parameter $M(L)$, (b) the magnetic susceptibility $\chi(L)$ at $T_{c}=6.6802$ for the finite-size lattices with $4 \leq L \leq 22$. 
in $d \geq 4$ has $\beta=1 / 2, \Upsilon=1$ and $\nu=1 / 2$, the new critical exponent $Q$ is 0.9904(16) for the order parameter and 1.0415(13) for the magnetic susceptibility (Table I, II). Both the order parameter and the magnetic susceptibility are 1.00 because $Q$ is equal to $d / d_{c}$ where $d_{c}=4$. Thus, their theoretical values are in good agreement with the values obtained from the data on the simulations.

In $d \geq 5$ dimensions, the Q-FSS relations used in the analysis are given below $[5,7,12,14]$ :

At $T=T_{c}$,

$$
M_{L}(t)=L^{\frac{-\beta Q}{\nu}} \propto L^{\frac{-d}{4}}
$$

and

$$
\chi_{L}(t)=L^{\frac{\gamma Q}{\nu}} \propto L^{\frac{d}{2}} .
$$

TABLE I

The theoretical values and the values obtained from the simulations of the Ising model on the Creutz cellular automaton for the new critical exponent $\mathbf{Q}$ in the dimensions $4 \leq d \leq 8$ for the order parameter.

\begin{tabular}{c|c|c|c}
\hline \hline Dimension $(d)$ & $\begin{array}{c}\frac{-\beta \mathbf{Q}}{\nu} \\
(\text { this work })\end{array}$ & $\begin{array}{c}\text { Koppa } \boldsymbol{Q} \\
(\text { this work }) \\
\left(\beta=\frac{1}{2}, \Upsilon=1,\right. \\
\left.\nu=\frac{1}{2}\right)\end{array}$ & $\begin{array}{c}\text { Theoretical } \\
\text { value } \\
\text { of koppa } \mathbf{Q} \\
\left(\mathbf{Q}=\frac{d}{d_{c}}\right)\end{array}$ \\
\hline 4 & $-0.9904(16)$ & $0.9904(16)$ & 1.00 \\
5 & $-1.2721(2)$ & $1.2721(2)$ & 1.25 \\
6 & $-1.4806(24)$ & $1.4806(24)$ & 1.50 \\
7 & $-1.7626(17)$ & $1.7626(17)$ & 1.75 \\
8 & $-1.9997(50)$ & $1.9997(50)$ & 2.00
\end{tabular}

TABLE II

The theoretical values and the values obtained from the simulations of the Ising model on the Creutz cellular automaton for the new critical exponent $Q$ in the dimensions $4 \leq d \leq 8$ for the magnetic susceptibility.

\begin{tabular}{c|c|c|c}
\hline \hline Dimension $(d)$ & $\begin{array}{c}\frac{\Upsilon \boldsymbol{Q}}{\nu} \\
\text { (this work) }\end{array}$ & $\begin{array}{c}\text { Koppa } \mathbf{Q} \\
(\text { this work }) \\
\left(\beta=\frac{1}{2}, \Upsilon=1,\right. \\
\left.\nu=\frac{1}{2}\right)\end{array}$ & $\begin{array}{c}\text { Theoretical } \\
\text { value } \\
\text { of koppa } \mathbf{Q} \\
\left(\mathbf{Q}=\frac{d}{d_{c}}\right)\end{array}$ \\
\hline 4 & $2.0830(13)$ & $1.0415(13)$ & 1.00 \\
5 & $2.5974(27)$ & $1.2987(27)$ & 1.25 \\
6 & $3.0265(1)$ & $1.5133(1)$ & 1.50 \\
7 & $3.5481(1)$ & $1.7741(1)$ & 1.75 \\
8 & $4.0265(28)$ & $2.0133(28)$ & 2.00
\end{tabular}

As previously done, the graphs of the data from the simulations are plotted by using Eq. (10) and Eq. (11) as seen in Fig. 2-5. The slopes of the graphs are considered to determine the new critical exponent $Q$. For the order parameter, $\mathbf{Q}=1.2721(2), \mathbf{Q}=1.4806(24)$, $Q=1.7626(17)$, and $Q=1.9997(50)$ when $d$ is at $d=5,6,7,8$, respectively (Table I). When studied on the magnetic susceptibility, the new critical exponents $Q$ are 1.2987(27), 1.5133(1), 1.7741(1), and 2.0133(28) in order of $d=5,6,7,8$ (Table II). At $d \geq 5$, the new critical exponent is also expected to be equal to $Q=d / 4$. Therefore, the theoretical values of $\mathrm{Q}$ are $\mathrm{Q}=1.25, \mathrm{Q}=1.50$,
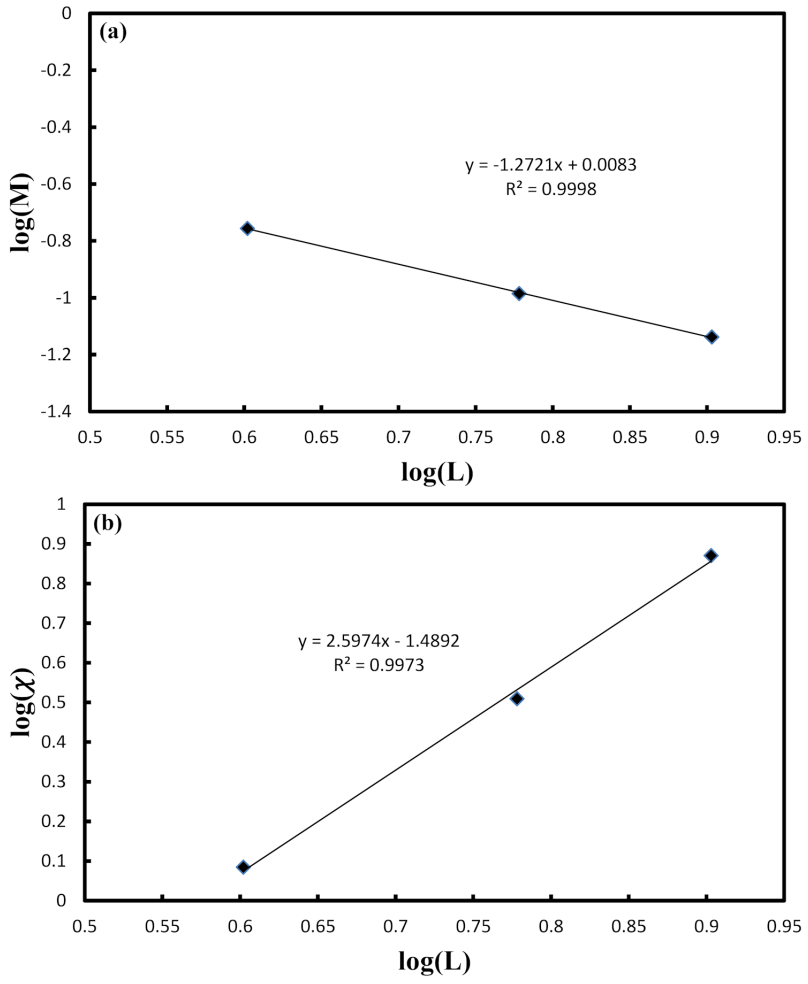

Fig. 2. The modified finite-size scaling (Q-FSS) for $d=5$ : (a) the order parameter $M(L)$, (b) the magnetic susceptibility $\chi(L)$ at $T_{c}=8.7787$ for the finite-size lattices with $4 \leq L \leq 8$.
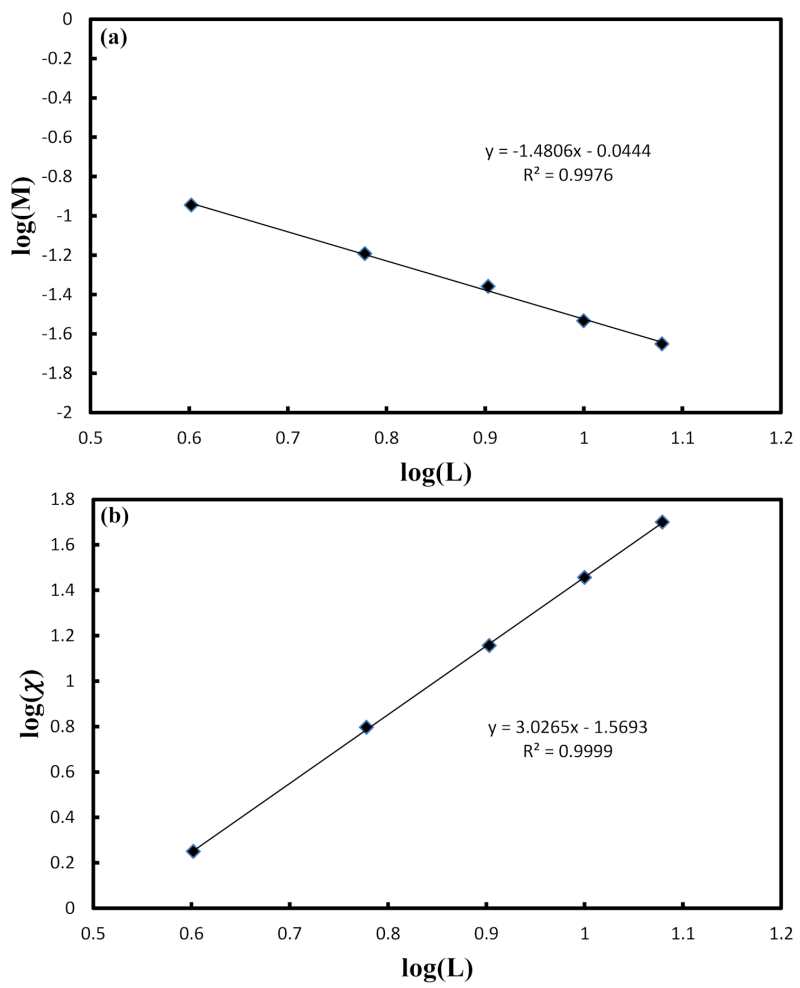

Fig. 3. The modified finite-size scaling (Q-FSS) for $d=6$ : (a) the order parameter $M(L)$, (b) the magnetic susceptibility $\chi(L)$ at $T_{c}=10.8348$ for the finite-size lattices with $4 \leq L \leq 12$. 

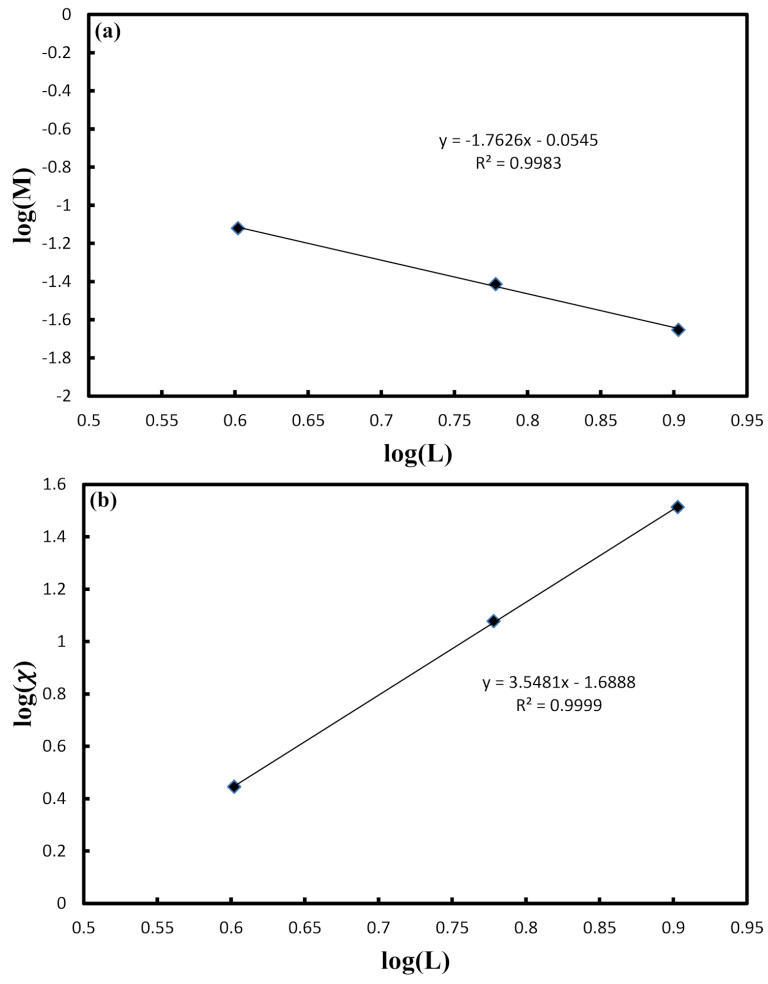

Fig. 4. The modified finite-size scaling (Q-FSS) for $d=7$ : (a) the order parameter $M(L)$, (b) the magnetic susceptibility $\chi(L)$ at $T_{c}=12.8690$ for the finite-size lattices with $4 \leq L \leq 8$.
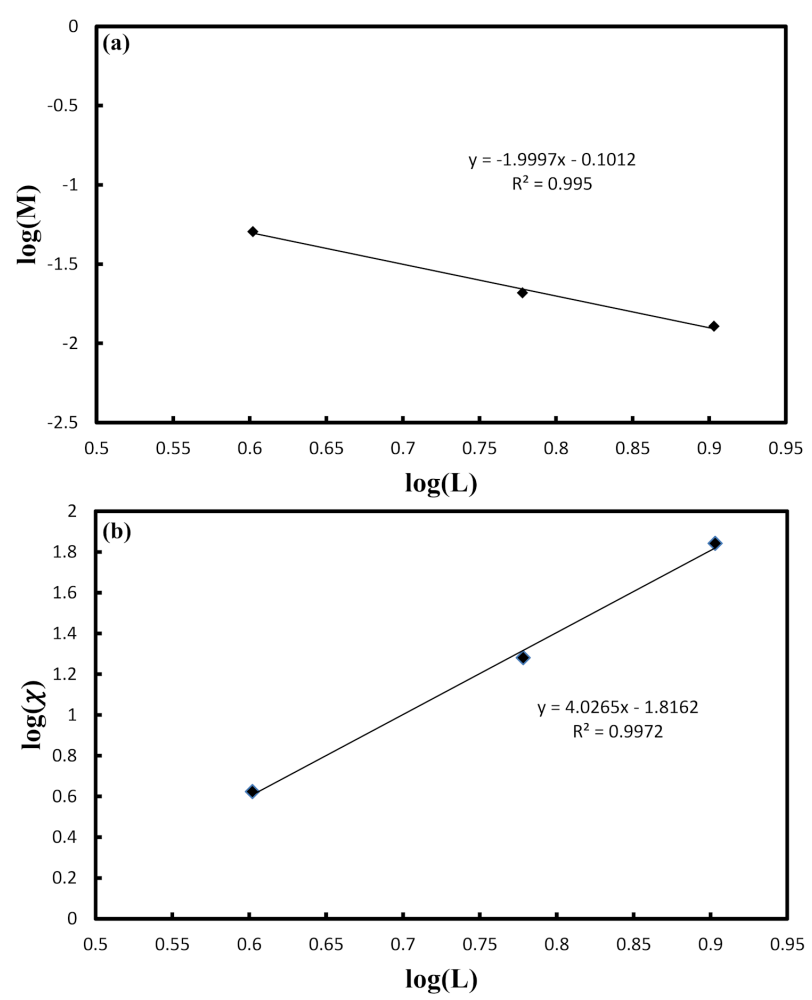

Fig. 5. The modified finite-size scaling (Q-FSS) for $d=8$ : (a) the order parameter $M(L)$, (b) the magnetic susceptibility $\chi(L)$ at $T_{c}=14.8920$ for the finite-size lattices with $4 \leq L \leq 8$.
$\mathbf{Q}=1.75$ and $\mathbf{Q}=2.00$ at $d=5,6,7,8$, respectively Table I, II. It is seen that the theoretical values and the values from the graphs agree with each other for both the order parameter and the magnetic susceptibility.

\section{Conclusions}

It is well known that standard FSS is universal at and below the upper critical dimension $d=d_{c}$ when hyperscaling holds and where the correlation length is comparable to the extent $L$ of a system $[15,16]$. Above $d_{c}$, the standard hyperscaling breaks down and dangerous irrelevant variables occur. Thus, standard FSS needs to be modified. At this point, there should be mentioned a new critical exponent, denoted by $Q$. Since it is universal, $Q$ has similar notation as the critical exponents $\alpha, \beta, \gamma$, $\delta, \eta$, and $\nu$, this notation was standardized by Fisher in the $1960 \mathrm{~s}[6]$. FSS is implemented by the substitution $t \rightarrow L^{-Q / \nu}$ instead of $t \rightarrow L^{-1 / \nu}$, and then this gives the modified FSS or Q-FSS. In Q-FSS relations, the new critical exponent $Q$ is equal to $d / d_{c}$.

To interpret the results of the new critical exponent $Q$ obtained from the simulations of the Ising model on the Creutz cellular automaton, all the results are in good agreement with its theoretical values. Certainly, there are errors when the estimated values of $\mathbf{Q}$ are compared to the theoretical values of $Q$ for each dimension as are in every study. This work shows that the new critical exponent $\mathbf{Q}$ is valid above the upper critical dimension.

\section{References}

[1] H.W.J. Blöte, R.H. Swendsen, Phys. Rev. B 22, 4481 (1980).

[2] M. Creutz, Ann. Phys. 167, 62 (1986).

[3] Z. Merdan, O. Karakus, Int. J. Theor. Phys. 55, 4822 (2016), Z. Merdan, N. Aras, C. Kurkcu, Acta Phys. Pol. A 129, (2016), Z. Artuc, Z. Merdan, S. Yesiltas, M.K. Ozturk, M. Tez, Physica A $\mathbf{4 8 6}$, 901 (2017).

[4] W.M. Lang, D. Stauffer, J. Phys. 20, 5413 (1987).

[5] E.J. Flores-Sola, B. Berche, R. Kenna, M. Weigel, Eur. Phys. J. B 88, 1 (2015).

[6] R. Kenna, B. Berche, Condens. Matter Phys. 16, 1 (2013).

[7] M.N. Barber, in: Phase Transitions and Critical Phenomena, Vol. 8, Eds. C. Domb, J.L. Lebowitz, Academic Press, New York 1983, p. 145.

[8] B. Berche, R. Kenna, J.-C. Walter, Nucl. Phys. B 865, 115 (2012).

[9] R. Kenna, B. Berche, Europhys. Lett. 105, (2014).

[10] N. Aktekin, in: Annual Rev. Comput. Phys., Vol. 7 , Ed. D. Stauffer, World Sci., 1999, p. 1.

[11] N. Aktekin, J. Stat. Phys. 104, 1397 (2001).

[12] Z. Merdan, R. Erdem, Phys. Lett. A 330, 403 (2004), Z. Merdan, A. Duran, D. Atille, G. Mulazimoglu, A. Gunen, Physica A 366, 265 (2006), M. Kalay, Z. Merdan, Mod. Phys. Lett. B 21, 1923 (2007). 
[13] Z. Merdan, C. Kurkcu, M.K. Ozturk, Low Temp. Phys. 40, 1360 (2014).

[14] E.J. Flores-Sola, B. Berche, R. Kenna, M. Weigel, Phys. Rev. Lett. 116, 115701 (2016).
[15] M.E. Fisher, in: Critical Phenomena. Proc. 51st Enrico Fermi Summer School, Varenna (Italy), 1971, Ed. M.S. Green, Academic Press, New York 1971, p. 1.

[16] M.E. Fisher, M.N. Barber, Phys. Rev. Lett. 28, 1516 (1972). 